\title{
Diagnostic laboratory testing for Charcot Marie Tooth disease (CMT): the spectrum of gene defects in Norwegian patients with CMT and its implications for future genetic test strategies
}

Rune Østern ${ }^{1,2^{*}}$, Toril Fagerheim¹, Helene Hjellnes ${ }^{1}$, Bjørn Nygård ${ }^{1}$, Svein I Mellgren ${ }^{2,3}$ and Øivind Nilssen ${ }^{1,2}$

\begin{abstract}
Background: Current genetic test algorithms for Charcot Marie Tooth (CMT) disease are based on family details and comprehensive clinical and neurophysiological data gathered under ideal conditions for clinical assessment. However, in a diagnostic laboratory setting relying on external test requisitions and patient samples, such conditions are not always met. Our objective was therefore to perform a retrospective evaluation of the data given in laboratory request forms and to assess their quality and applicability with regard to the recommended algorithms for CMT diagnostics. As we are the main test centre for CMT in Norway our results also provide an overview of the spectrum of gene defects in the Norwegian CMT population.
\end{abstract}

Methods: Genetic testing was performed according to polyneuropathy type; demyelinating/mixed: PMP22 duplication, MPZ, EGR2, LITAF, NEFL, PMP22, GJB1, axonal: MFN2, MPZ, NEFL, and GJB1.

Results: Diagnostic testing of index patients was requested in 435 of the 549 cases. Seventy-two (16.6\%) positive molecular genetic findings were made. The majority (94.6\%) of mutation positive cases showed disease onset before 50 years of age. PMP22 (duplication), MPZ, GJB1 and MFN2 mutations constituted $95.8 \%$ of the positive findings. Within the nerve conduction study groups, mutation detection rates were; demyelinating 33.8\%; mixed 29.0\%; axonal 8.8\%; unspecified 16.5\%.

Conclusion: We suggest a simplified algorithm intended for referral centres, dealing with DNA/blood samples, which involves the assessment of age at onset and neurophysiological data followed by testing of four genes; PMP22 (duplication), MPZ, GJB1 and MFN2. Patients negative for mutations in those four genes should be subjected to evaluation at an interdisciplinary inherited neuropathy clinic with the capacity for extended molecular genetic analysis by next generation sequencing.

Keywords: Charcot-Marie-Tooth, Genetic and inherited disorders, Neuromuscular diseases, Mutation analysis, Clinical neurophysiology, Guidelines

\footnotetext{
* Correspondence: Rune. Andre. Helland. Ostern@unn.no

'Department of Medical Genetics, University Hospital of North-Norway,

Tromsø NO9038, Norway

${ }^{2}$ Department of Clinical Medicine, Neuromuscular Research Group, University

of Troms $\varnothing$, Tromsø N09037, Norway

Full list of author information is available at the end of the article
} 


\section{Background}

Charcot Marie Tooth disease (CMT) is the most prevalent hereditary neuropathy [1]. In the population of Western-Norway the prevalence has been estimated to 41:100000 [2].

The classic clinical picture of Charcot Marie Tooth disease is characterized by muscular atrophy and weakness in the distal parts of the legs, absence of Achilles tendon reflexes, pes cavus, hammertoes and loss of touch and vibratory sensation. Nerve Conduction Velocity (NCV) in the motor median nerve is used to divide autosomal dominant CMT into CMT1 $(<38 \mathrm{~m} / \mathrm{s})$, CMT2 $(>38 \mathrm{~m} / \mathrm{s})$ [3] and dominant intermediate CMT $(25-45 \mathrm{~m} / \mathrm{s})$ [4]. Autosomal recessive CMT is called CMT4 and X-linked CMT CMTX independent of the NCV. Subtypes are defined by the mutant gene and more than $40 \mathrm{CMT}$ associated genetic loci have been identified [5]. The most common CMT subtypes are CMT1A due to a PMP22 duplication $(70-80 \%$ of the CMT1 cases) [6,7], CMT2A2 caused by MFN2 mutations $(10-30 \%$ of the CMT2 cases) [8,9], CMTX1 (GJB1) [4] and CMT4A (GDAP1). Atypical clinical presentations are well documented, particularly for CMT2 [10], CMT4, and CMTX [6]. The recessive CMT4 is associated with an early onset and severe symptoms.

Due to the clinical and genetic heterogeneity, the low sensitivity of genetic testing for CMT2 and scarcity of clinical data, CMT as a group represents a number of challenges for diagnostic laboratories. Recommended algorithms for CMT testing relies on exact clinical details, results from nerve conduction studies (NCS) and inheritance patterns gathered under ideal conditions $[11,12]$ and are therefore suited for specialized neuromuscular or inherited polyneuropathy clinics. Such conditions, however, rarely reflect the reality in everyday practice in laboratories dealing with samples from external patients and, thus, the yield of positive genetic test results in this group is significantly lower [13]. In Norway the vast majority of patients are being tested in this context and, likewise, in a recent UK study [13] almost 2/3rds of investigations were performed on CMT samples from external patients, demonstrating that this is not only a Norwegian phenomenon. In spite of the different test situations and the more heterogeneous nature of the latter group, in depth studies of the diagnostic efficacy of genetic testing of samples sent from external requisitioners are lacking. With regards to guidelines for testing, however, the two groups are often treated as if they were the same. Therefore, our main objective was to assess the quality and applicability of the recommended algorithms for CMT diagnostics in the diagnostic laboratory context. We have reviewed 559 requests for CMT testing received during the period from year 2004 to 2010 . We aimed at investigating to which extent available clinical and family information is influencing the success in the identification of disease causing variants. Based on these results we suggest a strategy for molecular genetic CMT testing for diagnostic laboratories which will provide an increase in conclusive test results, a decrease in false positive results and a more efficient use of resources. Furthermore, the results presented here may be of assistance in delineating the patient group that requires the services of an interdisciplinary inherited neuropathy clinic that have the capacity of detailed clinical and neurophysiological studies by experienced clinicians, and also extended molecular genetic analysis by next generation sequencing (NGS).

\section{Methods}

\section{Patient population}

The Department of Medical Genetics University Hospital of North-Norway is a part of the National Neuromuscular Centre and serves as a referral centre for genetic testing of patients with neuromuscular disorders from all parts of Norway. The annual number of samples received from patients with suspected CMT increased steadily from 25 in 2004 to 147 in 2010; accumulating at a total number of 559 for the seven-year period. Diagnostic, carrier or predictive, testing for known family mutations were requested for 87 patients. These results are not included in this work. Male-to-female ratio in the remaining 472 cases was 1.4:1, and mean age 47.4 $( \pm 21.7)$ years. Based on the information received in the laboratory request forms, 37 requests were rejected. Figure 1A shows the annual sample count and the proportion of rejected samples. The test algorithm was shortened with one or more of the requested analyses in 137/435 investigated index cases. An average of 2.7 genes pro sample were tested in those cases (Additional file 1: Table S1). The rejected group and those with deviation from the test protocol lacked information about nerve conduction studies (NCS) and had sparse or irrelevant clinical information.

\section{Genetic analyses}

DNA was extracted from peripheral blood cells using a Genovision M48 (Qiagen) or Biorobot EZ-1 (Qiagen) systems. Patients with demyelinating polyneuropathy were screened with a CMT1 test battery containing Multiplex Ligation dependent Probe Amplification (MLPA) of the PMP22 region for the assessment of quantitative alterations and by DNA sequencing of the MPZ, EGR2, LITAF, NEFL, PMP22 and GJB1 coding exons including at least $20 \mathrm{nt}$ of each flanking intron sequence. Cases with nerve conduction velocities in the intermediate range or with mixed polyneuropathy were also screened with the CMT1 test battery. Patients with axonal polyneuropathy were investigated using a CMT2 test battery which included DNA sequencing of the MFN2, MPZ, NEFL and 


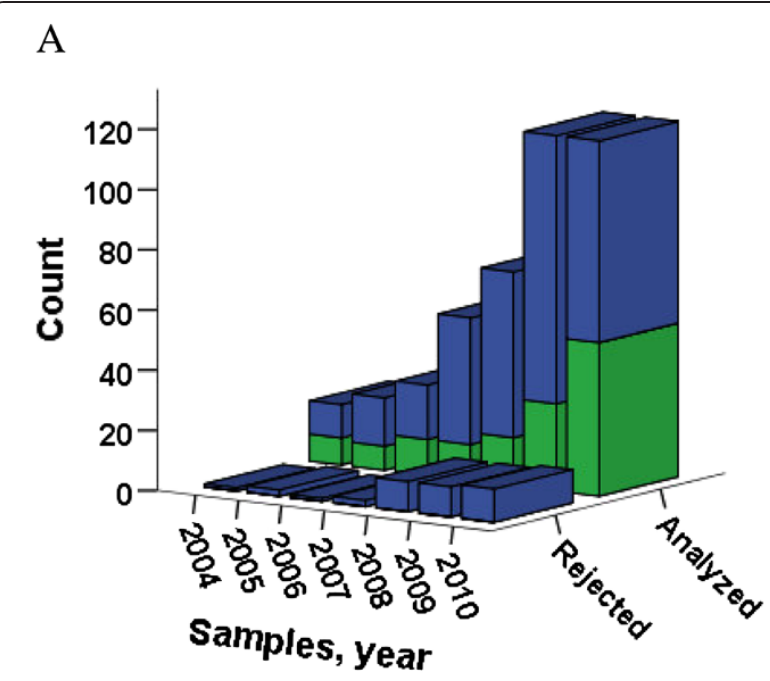

$\mathrm{C}$

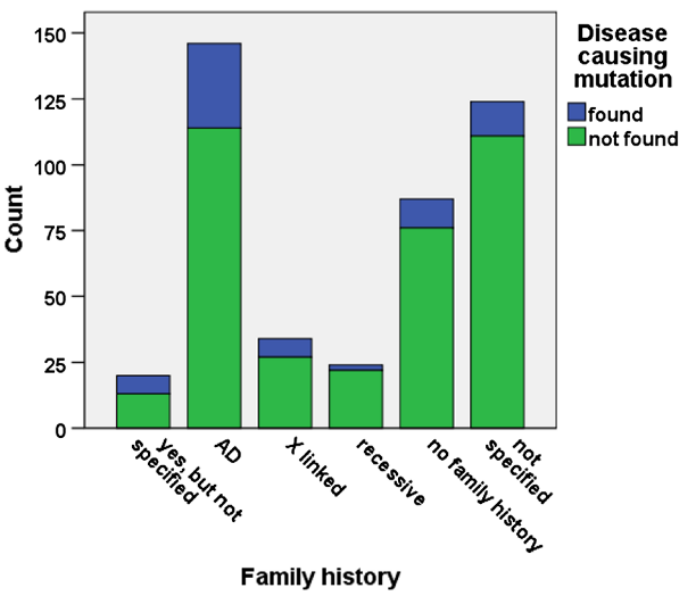

B

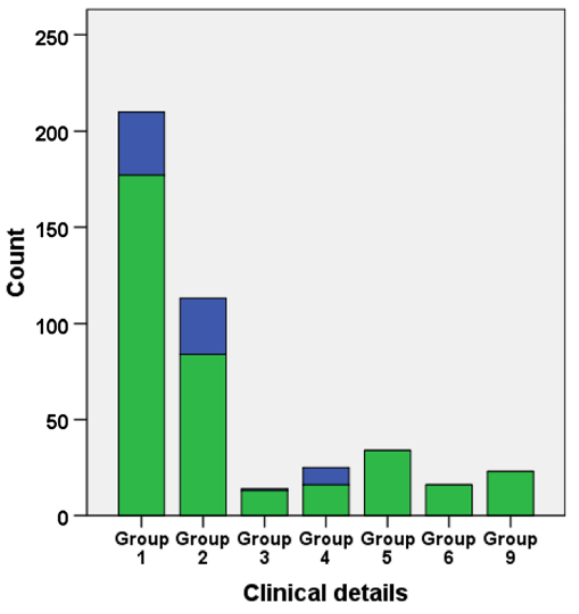

D

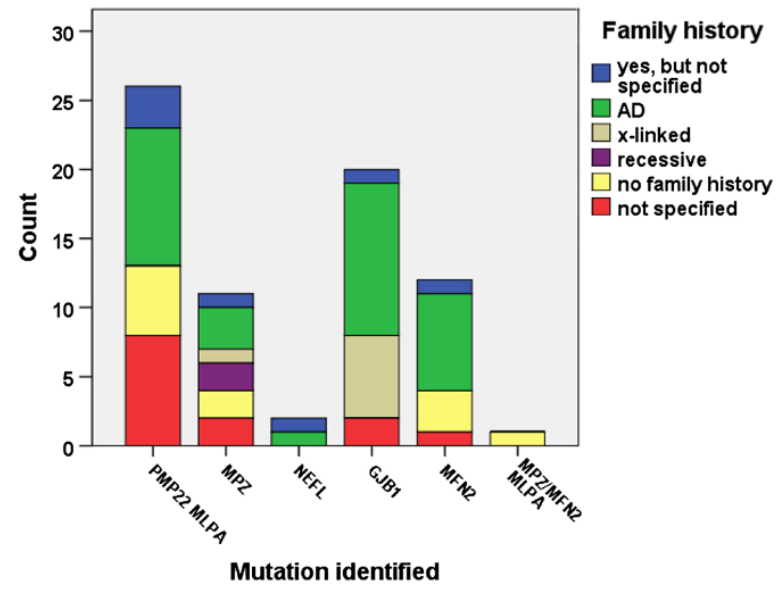

$\mathrm{E}$

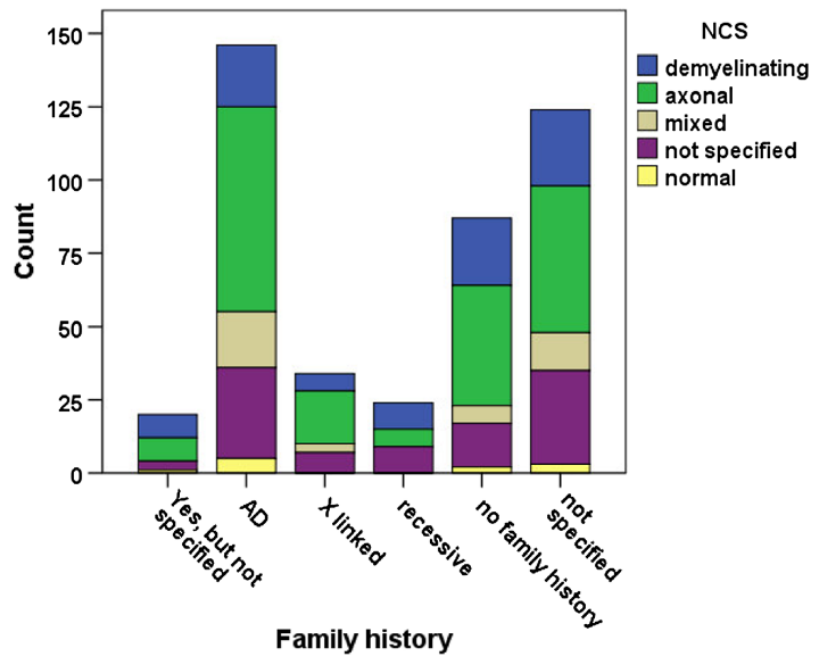

Figure 1 (See legend on next page.) 
(See figure on previous page.)

Figure 1 Recruitment of patient samples, patterns of inheritance, clinical details and genetic findings among the CMT patients. (A) The annual number of rejected and analyzed samples, 472 in total. Blue color; analyzed in accordance with protocol; green color; analyzed, but with deviation from protocol. (B) The sample count and number of findings in the individual clinical groups. 1; Polyneuropathy/CMT? No further information. 2; Specified symptoms of classical CMT. 3; As 2, but specified as severe. 4; As 2, but also with hearing impairment/deafness, pyramidal features, fasciculation, tremor, white matter changes on MRI. 5 Specified symptoms of atypical CMT (Additional file 1: Table S3). 6; Polyneuropathy as part of a more complex clinical picture with additional features usually not seen in association with CMT. 9; Requesting physician primarily suspects alternative diagnosis. (C) The sample count and number of findings in relation to mode of inheritance. (D) The patterns of inheritance in relation to genetic findings. (E) The NCS results in relation to pattern of inheritance.

GJB1 coding exons including at least $20 \mathrm{nt}$ of each flanking intron sequence. The group of patients displaying normal NCS results was analysed with the CMT2 test panel. Cases, in whom the polyneuropathy type could not be specified, were classified as "deviation from test protocol" if they were not analyzed with both the CMT1 and CMT2 panel. Based on clinical and family information, the GDAP1 gene was sequenced in individual cases (58/435) representing all polyneuropathy groups. MPZ/ MFN2 MLPA was performed on 229/435 samples.

The primers used for PCR amplification and DNA sequencing are listed in the Additional file 1: Table S2. MLPA reactions were performed using MLPA kits (MRC Holland, Amsterdam, The Netherlands), and PCR products were analyzed by fragment analysis using the Applied Biosystems 3130xl Genetic Analyzer. For CMT1A testing we used the SALSA MLPA KIT P033-B2 CMT1 and for the CMT1A/CMT2A2 testing SALSA MLPA P143 MFN2-MPZ probemix (further details are available upon request). Genetic variants were evaluated using the Alamut software (Interactive Biosoftware, San Diego, CA, USA) and in depth literature studies. The variants were categorized into five classes in accordance with the recommendations from the IARC Unclassified Genetic Variants Working Group [14]. Positive findings were defined as uncertain, probable or definite cause of the disease. Variants interpreted as non-pathogenic or likely non-pathogenic were defined as negative findings.

\section{Data collection, statistics and endpoint measures}

From the requisitions the following data were collected and systematized: The year the sample was received, the indication for testing (diagnostic, carrier/predictive or testing rejected), the specialty of the requesting physician (neurology, medical genetics, pediatrics, other), the age at onset of symptoms, the age at testing, whether or not supplemental information had been requested, results from motor NCV in the median nerve, whether or not there were deviations from the test algorithm, if CMT1A had been excluded previously at another laboratory, the type of polyneuropathy as indicated by NCS, the pattern of inheritance and a description of the family history, the number of affected relatives reported in addition to the index case, the gender of the index patient and their relatives, whether or not the mutation was found, and if yes, the name of the gene/mutation and the interpretation of its consequences (from 5 to 1 , Additional file 1: Table S3).

To investigate to which extent available clinical information is influencing the success of genetic diagnostics we found it necessary to stratify our patient population in 9 clinical categories as follows; 1: polyneuropathy/CMT - no further information; 2: Symptoms of classical CMT specified; 3: as 2, but more severe; 4 : as 2 , but with additional hearing impairment, pyramidal features, fasciculations, tremor or white matter changes on MRI; 5: Symptoms of atypical CMT, HSAN or HMN specified; 6: polyneuropathy with additional features not usually seen in association with CMT (typically ptosis, dysmorphic/multisystemic features); 7: healthy; 8: testing with regards to a family mutation, symptoms not specified; 9: requesting physician primarily suspects alternative diagnosis (CIDP, congenital myopathy, etc.). Further details, for each category, are shown in Additional file 1: Table S3.

Data from the individual categories were analyzed and data from the two patient groups, the mutation positive and the mutation negative, were compared. Statistical Package for the Social Sciences (SPSS) version 20.0 was used for the statistical analyses.

The research protocol was approved by the Regional Committee for Medical and Health Research Ethics for the counties of Nordland, Troms and Finnmark (REK Nord), and by the Norwegian Data Inspectorate. REK Nord specified that informed consent given by subjects/ next-of-kin was not necessary for this study. Procedures were in accordance with the 1964 Declaration of Helsinki and its later amendments.

\section{Results}

\section{Clinical information and mutation detection rate}

Among the 435 cases tested, 72 (16.6\%) were found to be mutation positive. They all belonged to the clinical groups 1-4 (Figure 1B). The 73 cases belonging to clinical group 5, 6 and 9 were all mutation negative. This difference in detection frequency between groups 1-4 and 5-9 is highly significant (Pearson chi-square 0.000). 
Clinical group 4 had the highest detection yield with $36 \%$ followed by group 2 (25.7\%), group 1 (15.7\%) and group 3 (7.1\%). The patients in the clinical group 4 had PMP22 duplications (4), point mutations in MPZ (2), NEFL (1), GJB1 (1) and a deletion of exon 13-17 in MFN2.

\section{Patterns of inheritance}

Inheritance patterns reported in the 435 cases were autosomal dominant (33.6\%), family history not specified (28.5\%), single case (20.0\%), X-linked (7.8\%), autosomal recessive $(5.5 \%)$ and positive, but with unspecified family history (4.6\%). Among the 72 cases with a positive genetic test result, information on family history was reported in $66.6 \%$. In the 363 cases with a negative test result, $48.5 \%$ had a positive family history (Pearson chi square $\mathrm{p}=0.005$ ). Positive test results were made in all groups with the highest yield in the group with positive, but unspecified family history (35.0\%), followed by the autosomal dominant (21.9\%), X-linked (20.6\%), single case (12.6\%), family history not specified (10.5\%) and autosomal recessive group (8.3\%) (Figure 1C). Seven of the patients with a positive test result had a suspected X-linked inheritance and 6 of these also had a mutation in the GJB1 gene (Figure 1D). Among 32 patients from families with a suspected dominant inheritance, but with no documented male to male transmission, 11 tested positive on mutations in the GJB1 gene. The two positive findings in the recessive group were "dominant mutations" in the $M P Z$ gene, but no "recessive mutations" were identified. Among 26 cases with PMP22 duplication, only 10 had been assigned as dominant inheritance. In comparison, $55.0 \%$ (11) of the patients with a GJB1 mutation had been suspected to come from families where CMT segregated as a dominant trait. In 231/435 samples information about the inheritance could not be used to specify CMT subtype because they were either single cases or positive, but with unspecified or unavailable family history. In addition, 47 cases with suspected autosomal dominant, X-linked and recessive inheritance, lacked results of Nerve Conduction Studies (NCS) (Figure 1E).

\section{Description of the family history}

The number of affected relatives was reported for 199 cases. Among 43 patients with a positive genetic test result the mean number of relatives specified was $2.14 \pm 1.28$. Among 156 patients with a negative genetic test result the mean number of relatives specified was $1.78 \pm 0.97$. Among the same 199 cases the gender of index and relatives were male and female in 119 cases (59.8\%), male only in $53(26.6 \%)$ and female only in 27 cases (13.6\%).

\section{Nerve conduction studies}

Polyneuropathy was reported as demyelinating in $21.4 \%$, axonal in $44.4 \%$, mixed in $9.4 \%$, not specified in $22.3 \%$, and normal in $2.5 \%$. The demyelinating and mixed polyneuropathy groups were analyzed with the same testing panel and showed equivalent mutation detection rates (33.8\%; 29.3\%), (Table 1). Patients, for whom NCS results suggested axonal neuropathy, constituted the largest group and showed the lowest detection rate (8.8\%). For patients with NCS-unspecified we obtained a detection rate of $16.5 \%$, similar to the rate found in the material as a whole (16.6\%). CMT1A had already been excluded at other laboratories in 59 cases. The mutation detection rate among these 59 cases was 22.0\% (3 MPZ, 1 NEFL, 7 GJB1 and 2 MFN2 mutations). The distribution of mutant genes within the NCS groups is similar to what has been reported in the literature. Independent of gender, patients with GJB1 mutations are represented in all polyneuropathy groups with the majority, however, in the mixed category.

\section{Age at disease onset and age at testing}

Figure 2A shows the bimodal distribution of age at testing in the group of 435 patients with a second peak starting at 40 years. The age distribution among the NCS groups at testing shows an early peak in the demyelinating group and a late peak in the axonal group, but also in the unspecified group (Figure 2B). Among the 72 cases with a positive genetic test result, the mean age at testing was $37.6 \pm 19.4$ years, and among the cases with a negative test result it was $48.9 \pm 21.6$ years. Almost half of the samples received (231/435) were from patients older than 50 years and 23 positive test results

Table 1 Mutation detection rate in the NCS groups

\begin{tabular}{llll}
\hline $\begin{array}{l}\text { Reported } \\
\text { result on } \\
\text { NCS }\end{array}$ & \multicolumn{2}{c}{ Disease causing mutation } & $\begin{array}{l}\text { Total } \\
\text { count }\end{array}$ \\
\cline { 2 - 3 } Demyelinating & 27 & Not found & \\
\hline & $(24)$ & 66 & 93 \\
& $29,0 \%$ & $(47)$ & \\
Axonal & $(33.8 \%)$ & $71,0 \%$ & \\
& 17 & $(66.2 \%)$ & 193 \\
Mixed & $8,8 \%$ & 176 & \\
& 12 & $91,2 \%$ & 41 \\
Not specified & $29,3 \%$ & 29 & \\
Normal & 16 & $70,7 \%$ & 97 \\
& $16,5 \%$ & 81 & 11 \\
Total & 0 & $83,5 \%$ & 435 \\
& $0 \%$ & 11 & \\
\hline
\end{tabular}

In 59 cases CMT1A had already been excluded at other laboratories. The results reported in brackets concern the demyelinating group excluding these cases. The NCS-normal group was analyzed with the CMT2 test panel. 
A

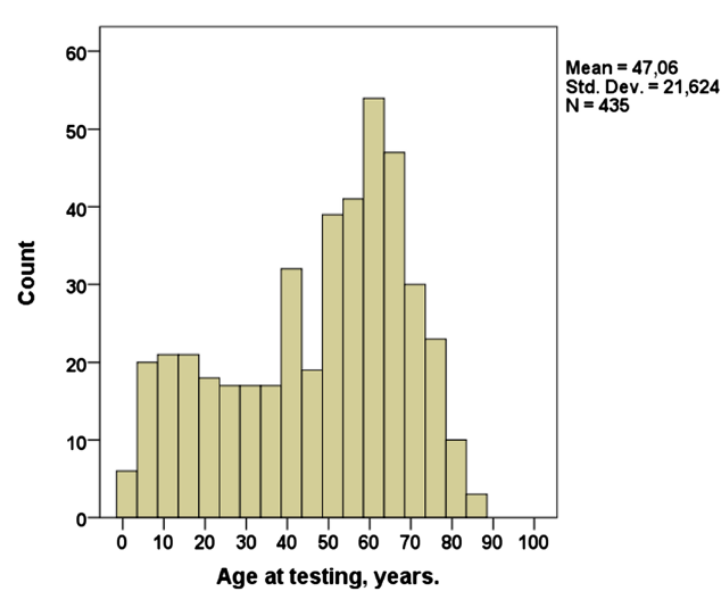

C

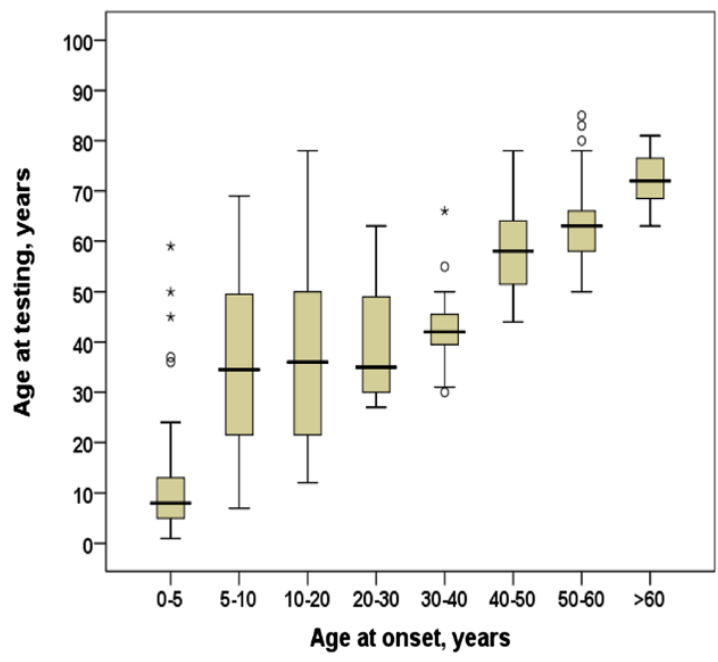

E

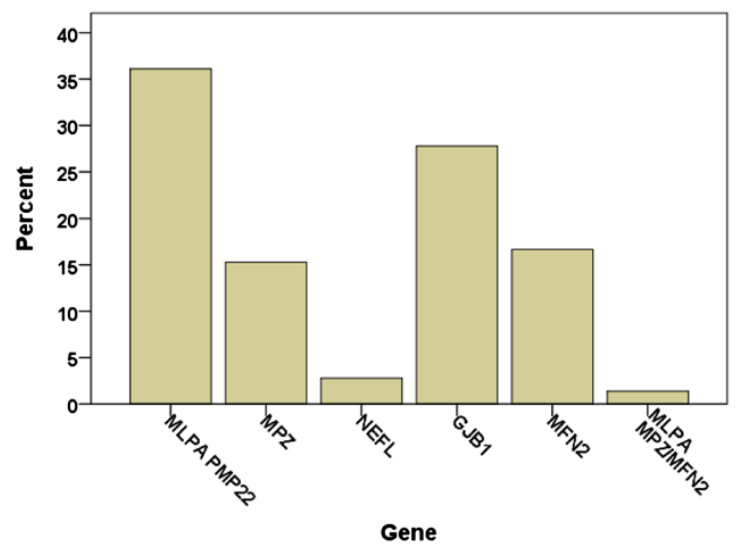

B

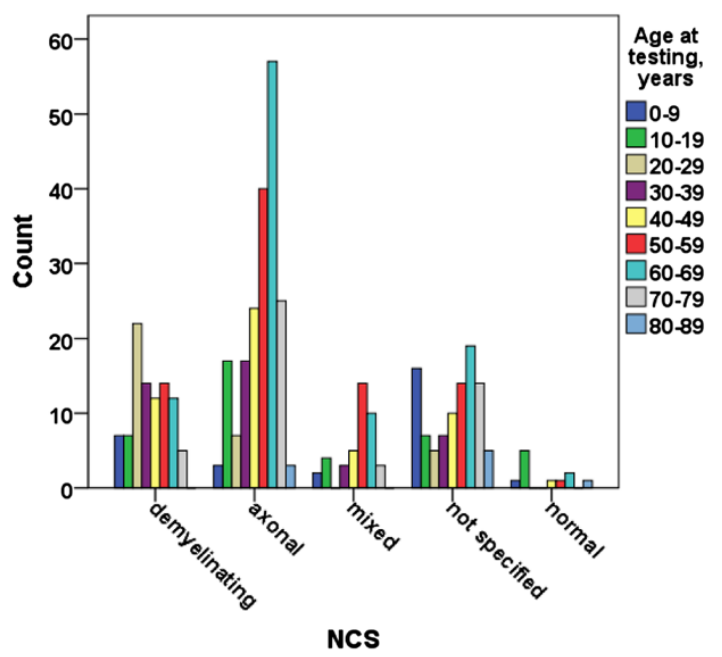

D

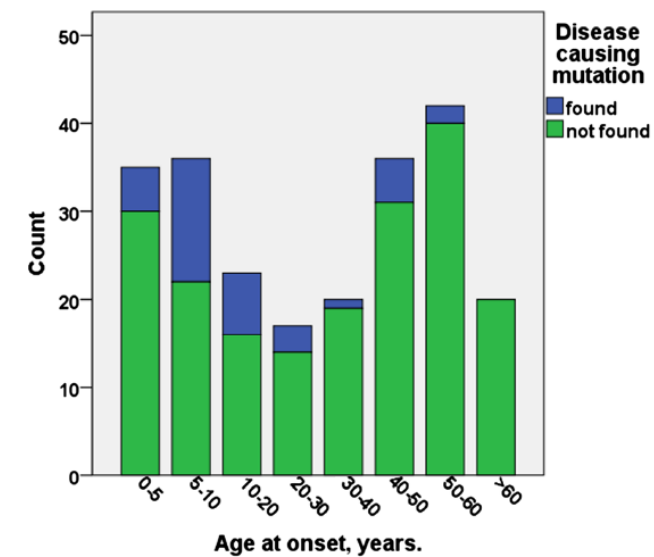

F

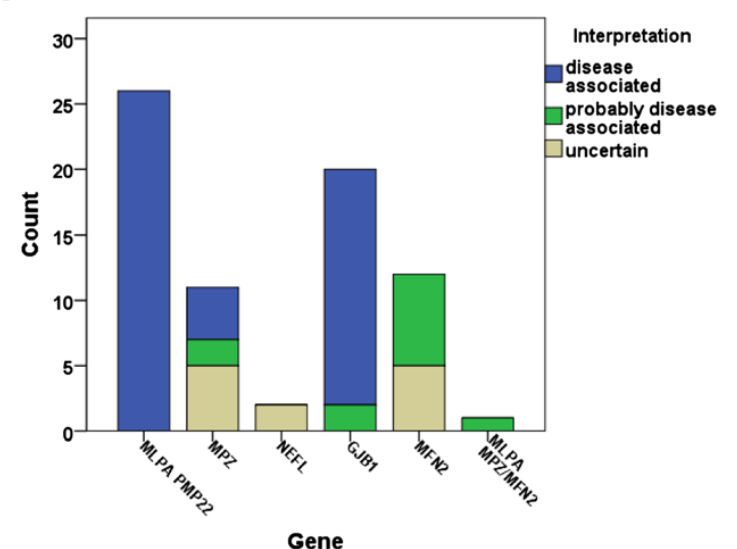

Figure $\mathbf{2}$ (See legend on next page.) 
(See figure on previous page.)

Figure 2 Clinical, NCS and genetic findings among the CMT patients. (A) Histogram; the age at testing in 435 patients. (B) The distribution of age at testing within the NCS groups. (C) Boxplot: the relationship between age at onset and age at testing in 229 samples. (D) The distribution of age at disease onset (229 cases) in the group with positive (blue) and negative (green) findings. (E) The relative frequency of genetic findings. (F) The distribution of genetic findings and interpretation of disease association for 72 mutations.

were made in this group. Although the differences in the detection yield obtained in the groups younger versus older than 50 years at testing is significant (Pearson chisquare 0.000 ), the association between age at onset and age at testing is loose (Figure $2 \mathrm{C}$ ).

Age of onset was reported for 229/435 cases (52.6\%). Among these, 37 (16.6\%) were found to be positive on genetic testing. Figure 2D compares the age at disease onset in the groups with a positive and negative test result. Most positive findings were made in patients with onset age $<30$ years $(78.3 \%)$ and the highest frequency was associated with onset between 5-20 years (56.7\%). The remaining fraction (21.7\%) of the positive findings was made in patients with reported onset between 30 and 60 years. However, in the patient material as a whole, $94.6 \%$ of the mutation positive patients showed disease onset before 50 years of age. Two mutations were found in the group with onset between 50 and 60 years: a duplication of the PMP22 region and a missense mutation of uncertain significance (p.Asp35Asn) in the $M P Z$ gene.

\section{Medical specialties and requisitioning}

Only index cases were included in this work. All 472 molecular genetic tests were requested by medical doctors. The vast majority was requested from neurology departments $(77.1 \%)$, but also from departments of medical genetics (7.4\%) and pediatrics (10.8\%). The remaining $4.7 \%$ of the test requests came from medical doctors belonging to other fields of medicine, mostly primary care. Medical genetic departments and "other" often cooperated with a neurologist or pediatrician when they requested testing. Due to sparse clinical information, supplementary information was asked for in 157 cases prior to the selection of testing strategy. The response rate was $61.8 \%$ (97/157). The additional information received was not always relevant, particularly when the questions were concerning NCS.

\section{Molecular genetic findings}

Figure $2 \mathrm{E}$ shows the relative proportion of positive test results for the various genes tested. The genetic variants detected were interpreted as definitely disease causing in 48, likely disease causing in 12 and uncertain in 12 cases (Figure 2F). Novel mutations not listed in The Human Gene Mutation Database Professional (HGMDp) (www. biobase-international.com) version 2013.2, are listed in Table 2. Genetic variants interpreted as likely not, or definitely not disease causing are not dealt with in the present article. The majority $(95.8 \%, 69 / 72)$ of the positive molecular genetic findings were either duplication of the $P M P 22$ region or sequence variants in either one of the MPZ, GJB1 or MFN2 genes. Testing with MPZ/ MFN2 MLPA was performed on 229 samples from all NCS groups and there was one positive finding (deletion of the MFN2 gene) in a patient with axonal

Table 2 Novel sequence variants not reported in the HGMDp database ${ }^{(t)}$

\begin{tabular}{|c|c|c|c|c|c|}
\hline Gene & CDNA & Protein & Type & het/homos & Classification $^{f}$ \\
\hline \multirow[t]{3}{*}{$M P Z$} & c. $679 \mathrm{~A}>\mathrm{T}$ & p.Arg227* & Nonsense & het & 4 \\
\hline & c. $368 \mathrm{G}>\mathrm{T}$ & p.Gly123Val & Missense & het & 3 \\
\hline & $\mathrm{C} .410 \mathrm{G}>\mathrm{A}$ & p.Gly137Asp & Missense & het & 3 \\
\hline NEFL & c.1027_1029del & p.Asp343del & In-frame deletion & het & 3 \\
\hline GJB1 & c.775del & p.Leu259* & Deletion & hemi & 4 \\
\hline \multirow[t]{6}{*}{ MFN2 } & c.2146_2148dup & p.Ala716dup & In-frame duplication & het & 3 \\
\hline & $c .250 A>G$ & p.Lys84Glu & Missense & het & 3 \\
\hline & c. $612 \mathrm{~T}>\mathrm{A}$ & p.Asp204Glu & Missense & het & 3 \\
\hline & c.653 T > C & p.Leu218Pro & Missense & het & 4 \\
\hline & c.692 C > T & p.Ser231Phe & Missense & het & 3 \\
\hline & C.1921 T > C & p.Tyr641His & Missense & het & 3 \\
\hline
\end{tabular}

${ }^{\dagger} \mathrm{HGMDp}$ version 2013.2.

\$het = heterozygous; homo = homozygous; hemi = hemizygous.

${ }^{\mathrm{E}}$ Classification of genetic variants in accordance with the recommendations from the IARC Unclassified Genetic Variants Working Group [14]; $4=$ probably disease causing, 3 = variant of uncertain significance. 
polyneuropathy while the remaining two findings were sequence variants of uncertain significance in the NEFL gene. Among the 116 cases analysed with regard to LITAF/SIMPLE and EGR2 mutations, and among the 196 cases sequenced with regards to $P M P 22$ point mutations, no disease causing mutations were found.

\section{Discussion}

All positive genetic test results were made on samples belonging to the clinical groups $1-4$, displaying the classical symptoms of CMT. Almost half of these were detected in clinical group 1 in which there were sparse, but relevant, clinical information. No disease causing mutations were found in patients for whom the clinical information gave reason to suspect atypical CMT2, or alternative diagnoses like hereditary sensory and autonomic neuropathy (HSAN), hereditary motor neuronopathy (HMN) or polyneuropathy as part of a more complex clinical picture. The low detection yield in clinical group 3 and in those with a recessive family history, as well as the absence of GDAP1 mutations in 58 selected patients, imply that we still know little about the genetics of severe/ recessive CMT in Norway. None of the patients with a MFN2 mutation had optic atrophy nor were they categorized as very severely affected.
We find that although a positive family history is associated with an increased probability of positive test results, the apparent pattern of inheritance is of limited value in the selection of testing strategy in most cases. The exceptions are cases with documented male-to-male transmission, leaving GJB1 testing redundant, as well as for cases where family history suggests a recessive inheritance, indicating that a causative mutation(s) is likely to be found outside the four common genes. However, the family history and subsequent segregation studies are very useful in evaluating genetic variants of unknown or doubtful significance.

Notably, in this study $63.9 \%$ of the cases could not be classified in accordance with CMT subtype definitions as they were single cases, cases with positive but unspecified family history, cases where family history was not mentioned, or more rarely because they lacked suitable NCS results.

The reported frequency of PMP22 duplication in index patients with suspected CMT1 ranges between 23.3\% and $60.7 \%$ (average $41.8 \%$ ) across different studies, and in different populations (Table 3) $[8,9,15-23]$. The reported frequency of $M P Z$ mutations ranges between $2.4 \%$ and $13.0 \%$ (average 5.0\%) and GJB1 mutations between $5.5 \%$ and $25.8 \%$ (average $8.8 \%$ ) (Table 3 ). The mutation detection yields reported in different studies

Table 3 Mutation detection rates associated with suspected HMSN in various populations

\begin{tabular}{|c|c|c|c|c|c|c|c|c|c|c|c|c|c|c|}
\hline \multirow[t]{3}{*}{ Population } & \multirow[t]{3}{*}{$N$ total } & \multicolumn{8}{|c|}{ Demyelinating/mixed polyneuropathy $\left(\mathrm{CMT1}, \mathrm{DSS}^{\dagger}, \mathrm{CHN}^{\$}\right)$} & \multicolumn{5}{|c|}{ Axonal polyneuropathy (CMT2) } \\
\hline & & \multirow[t]{2}{*}{$\mathbf{N}$} & \multicolumn{7}{|c|}{ (\%) } & \multirow[t]{2}{*}{$\mathbf{N}$} & \multicolumn{4}{|c|}{ (\%) } \\
\hline & & & PMP22(dup) & $M P Z$ & EGR2 & LITAF & NEFL & PMP22 & GJB1 & & MFN2 & NEFL & $M P Z$ & GJB1 \\
\hline Spanish [15] & 47 & 35 & excl & 8.6 & - & - & - & 2.9 & 17.1 & 7 & - & - & 0 & 57.1 \\
\hline Korean [16] & 57 & 32 & 46,9 & 3.1 & 3.1 & - & 3.1 & 3.1 & 6.2 & 18 & - & 5.6 & 11.1 & 5.6 \\
\hline Italian [17] & 172 & 170 & 57.6 & 2.4 & 0 & - & - & 1.2 & 7.1 & 0 & - & - & - & - \\
\hline Australian [18] & 224 & $224^{f}$ & 60.7 & 3.1 & - & - & - & 1.3 & 12.1 & 0 & - & - & - & - \\
\hline Finnish [19] & 58 & 23 & excl & 13.0 & - & - & - & 4.3 & 13.0 & 29 & - & - & 0 & 24.1 \\
\hline Japanese [20] & 354 & 227 & 23.3 & 8.8 & 0.4 & 0 & 3.5 & 4.4 & 8.4 & 127 & 11.3 & 0 & 3.9 & 4.7 \\
\hline British [21] & 775 & 443 & 28.2 & 4.2 & 3.3 & - & 9.5 & 5.3 & 25.8 & $N R$ & 13.6 & - & 4.6 & 2.7 \\
\hline American [6] & 153 & 145 & 51.6 & 3.4 & 0.7 & - & 0 & 3.4 & $5.5^{*}$ & 7 & - & 14.3 & 0 & 42.9 \\
\hline Russian [22] & 174 & 108 & 53.7 & 4.6 & - & - & - & 1.9 & 7.4 & 32 & - & - & 0 & 3.1 \\
\hline European [9] & 323 & 26 & - & - & - & - & - & - & - & 249 & 11.2 & - & - & - \\
\hline American [8] & 13 & 0 & - & - & - & - & - & - & - & 13 & 23.1 & - & - & - \\
\hline American [23] & 39 & 1 & - & - & - & - & - & - & - & 38 & 17,9 & - & - & - \\
\hline Average & 2389 & 1407 & 41.8 & 5.0 & 0.5 & 0 & 2.2 & 2.6 & 8.8 & 520 & 12.2 & 1.3 & 3.2 & 10.0 \\
\hline This study & 435 & 134 & $18.7^{\ddagger}$ & 6.0 & 0 & 0 & 0.7 & 0 & 6.7 & 193 & 5.7 & 0.5 & 1.0 & 1.5 \\
\hline
\end{tabular}

The analyses were performed at referral centres for external patients (prospective testing of index cases). The discrepancy between $\mathrm{N}$ total and $\mathrm{N}$ CMT1/CMT2 are due to additional categories in the studies not listed in the table. - Data not available.

${ }^{\dagger}$ Dejerine-Sottas syndrome.

${ }^{\$}$ Congenital hypomyelinating neuropathy.

${ }^{\mathrm{E}} \mathrm{NCV}<50 \mathrm{~m} / \mathrm{s}$.

" $" \mathrm{CMTX".}$

${ }^{\ddagger}$ 20/107, in 27 cases CMT1A were excluded at another laboratory. 
are not directly comparable. This is due to differences in sampling methods, testing strategies and ways of reporting the results. Also, most reports only give a detailed description of patients with positive test results, not the entire cohort. The frequency of PMP22 duplications among the demyelinating polyneuropathy patients in this material was lower than average (18.7\%), whereas the frequency of MPZ mutations (6.0\%) and GJB1 mutations (6.7\%) were close to average. This may indicate that PMP22 duplications are less frequent or that a large part of the families with PMP22 duplication in Norway already were identified by other laboratories before the start of our observation period. Also, a prior exclusion of CMT1A might have been underreported in the patient request forms. A previous study, however, estimated a similar frequency of the PMP22 duplication (13.6\%) in families with CMT in a population in Southern Norway (inhabitants of Akershus County) [24]. The rate of positive molecular diagnostic findings in CMT patients attending neuropathy clinics, compared to external samples sent for molecular genetic investigation, are significantly different. The rate of positive findings in the neuropathy clinic group [12,13] are similar to the high detection rates documented in the literature $[6,7,9]$, whereas the frequencies found in diagnostic laboratories are lower as shown in this and other studies [13, Table 3].

Interestingly, in patients for whom NCS were lacking we obtained a mutation detection rate of $16.5 \%$, similar to the detection rate found in the patient material as a whole (16.6\%). This demonstrates that the lack of NCS results should not be used as an absolute exclusion criterion for genetic testing. However, the laboratory offered a wider panel (for both demyelinating and axonal CMT) in those who did not have NCS results specified, hence spending more resources.

The association between age at onset and age at testing is loose. A strong rise in incoming samples from patients of high age, mainly due to a rise in the requested tests for patients with axonal polyneuropathy, is associated with an increased number of phenocopies with axonal polyneuropathy of different genesis. Moreover, on average $42.9 \%$ of the patients $>50$ years at testing have a negative or unspecified family history, further contributing to this tendency. Age at onset is therefore one of the most important parameters in the decision algorithm. Unfortunately, age at onset was reported only in $52.6 \%$ of the cases presented here.

The majority (95.8\%) of the positive molecular genetic findings were either duplication of the PMP22 region or a sequence variant in either one of the $M P Z, G J B 1$ or MFN2 genes. The present study strongly indicates that none of the genes that have been reported elsewhere to be involved in rare cases of CMT (LITAF/SIMPLE, EGR2, PMP22, NEFL, GDAP1 and MPZ/MFN2 deletions/ duplications) are commonly involved in CMT in the Norwegian population. These results are in accordance with other recent publications on the frequency of genetic subtypes $[12,13]$ stating that $>90 \%$ of mutations in individuals with a positive genetic test are caused by $P M P 22$, GJB1, MFN2 and MPZ, and that "recessive mutations" are rare. "Recessive mutations" in $S H 3 T C 2$ have been reported as a frequent cause of demyelinating CMT in some populations [25]. SH3TC2 was not a part of the gene panel used in this study, but in a recent paper the frequency was reported to be $0.3 \%$ in a large CMT cohort from the United Kingdom [13].

\section{Conclusion}

We suggest a pragmatic two-tier approach to genetic testing in CMT; the first tier should be used by referral centres receiving blood or DNA samples, not patients. Genetic tests in this setting should primarily be selected on the basis of nerve conduction studies. Patients showing demyelinating or mixed polyneuropathy/intermediate nerve conduction velocities, and those in whom the polyneuropathy type is uncertain, should be tested with PMP22 MLPA and DNA sequencing of the MPZ and GJB1 genes. In cases with documented axonal polyneuropathy DNA sequencing of $M F N 2, M P Z$ and GJB1 is recommended. A similar conclusion was recently made, based on a study of a CMT population from the UK [13]. Unless there is a clear clinical indication of CMT and positive family history, fifty years at age of onset seems an appropriate cut off age for which testing should be rejected. Good clinical documentation is increasing the probability of detecting disease causing mutations. For cases with sparse clinical information, limited genetic testing as suggested above should be carried out provided that the requests come from experienced neurologists, geneticists or paediatricians. Tests should be restricted to cases with classical CMT, with or without additional features as described in the clinical group 4 .

The second tier should be based on interdisciplinary investigation of patients at neuromuscular centres. Several excellent algorithms exist for this purpose [11,12]. Powerful tools, like next generation sequencing (NGS), are nowadays implemented in clinical practice and provide possibilities for more efficient genetic diagnostic service to patients with hereditary polyneuropathies [26]. Of note, we did not analyse all genes known to be associated with CMT. A broad scanning of CMT associated genes likely would have increased the mutation detection rate. Patients negative for mutations in PMP22, GJB1 and MPZ, and patients with atypical CMT2, HSAN and HMN should be subjected to extended analysis by NGS and prioritization of the genes to be investigated should be based on detailed studies of the phenotype. 


\section{Additional file}

Additional file 1: Table S1. Tests performed on the 137 samples deviating from the test algorithm. Table S2. Mutation analysis was carried out by sequencing of all coding regions and their exon-intron boundaries using the listed primers. ' 3 ' untranslated region of the gene. Table S3. The variables used in the study. 'variable not relevant for this paper.

\section{Competing interests}

Potential conflicts of interest; none.

\section{Authors' contributions}

$R \varnothing$ has taken part in all stages of the project including design and conceptualization of the study, analysis and interpretation of the data and also in drafting and revising of the manuscript for intellectual content. TF has taken part in analysis and interpretation of the data and also in revising of the manuscript for intellectual content. HH has taken part in analysis and interpretation of the data and in revising of the manuscript for intellectual content. BN has taken part in analysis and interpretation of the data and in revising of the manuscript for intellectual content. SIM has taken part in all stages of the project including design and conceptualization of the study, analysis and interpretation of the data and also in drafting and revising of the manuscript for intellectual content. $\varnothing \mathrm{N}$ has taken part in all stages of the project including design and conceptualization of the study, analysis and interpretation of the data and also in drafting and revising of the manuscript for intellectual content. All authors read and approved the final manuscript.

\section{Acknowledgements}

This work was supported by The Norwegian Research Council Grant \# 199372.

\section{Author details}

'Department of Medical Genetics, University Hospital of North-Norway, Tromsø N09038, Norway. ${ }^{2}$ Department of Clinical Medicine, Neuromuscular Research Group, University of Tromsø, Tromsø N09037, Norway. ${ }^{3}$ Department of Neurology, University Hospital of North-Norway, Tromsø NO9038, Norway.

Received: 27 April 2013 Accepted: 17 September 2013 Published: 21 September 2013

\section{References}

1. Dyck PJ, Oviatt KF, Lambert EH: Intensive evaluation of referred unclassified neuropathies yields improved diagnosis. Ann Neurol 1981, 10:222-226

2. Skre H: Genetic and clinical aspects of Charcot-Marie-Tooth's disease. Clin Genet 1974, 6:98-118.

3. Harding AE, Thomas PK: The clinical features of hereditary motor and sensory neuropathy types I and II. Brain 1980, 103:259-280.

4. Nicholson G, Myers S: Intermediate forms of Charcot-Marie-Tooth neuropathy: a review. Neuromolecular Med 2006, 8:123-130.

5. Bird TD: Charcot-Marie-Tooth Hereditary Neuropathy Overview. Gene Reviews [http://www.ncbi.nlm.nih.gov/books/NBK1358/] (accessed June 02, 2012); 2012.

6. Boerkoel CF, Takashima H, Garcia CA, Olney RK, Johnson J, Berry K, Russo P, Kennedy S, Teebi AS, Scavina M, Williams LL, Mancias P, Butler IJ, Krajewski K, Shy M, Lupski JR: Charcot-Marie-Tooth disease and related neuropathies: mutation distribution and genotype-phenotype correlation. Ann Neurol 2002, 51:190-201.

7. Nelis E, Van Broeckhoven C, De Jonghe P, Löfgren A, Vandenberghe A Latour P, Le Guern E, Brice A, Mostacciuolo ML, Schiavon F, Palau F, Bort S, Upadhyaya M, Rocchi M, Archidiacono N, Mandich P, Bellone E, Silander K, Savontaus ML, Navon R, Goldberg-Stern H, Estivill X, Volpini V, Friedl W, Gal $A$, et al: Estimation of the mutation frequencies in Charcot-Marie-Tooth disease type 1 and heriditary neuropathy with liability to pressure palsies: a European collaborative study. Eur J Hum Genet 1996, 4:25-33.

8. Lawson VH, Graham BV, Flanigan KM: Clinical and electrophysiologic features of CMT2A with mutations in the mitofusin 2 gene. Neurology 2005, 65:197-204
9. Verhoeven K, Claeys KG, Züchner S, Schröder JM, Weis J, Ceuterick C, Jordanova A, Nelis E, De Vriendt E, Van Hul M, Seeman P, Mazanec R, Saifi GM, Szigeti K, Mancias P, Butler IJ, Kochanski A, Ryniewicz B, De Bleecker J, Van den Bergh P, Verellen C, Van Coster R, Goemans N, Auer-Grumbach M, Robberecht W, Milic Rasic V, Nevo Y, Tournev I, Guergueltcheva V, Roelens F, et al: MFN2 mutation distribution and genotype/phenotype correlation in Charcot-Marie-Tooth type 2. Brain 2006, 129:2093-2102.

10. Bienfait HM, Baas F, Koelman JH, de Haan RJ, van Engelen BG, Gabreëls-Festen AA, de Visser BW O, Meggouh F, Weterman MA, De Jonghe P, Timmerman V, de Visser M: Phenotype of CharcotMarie-Tooth disease Type 2. Neurology 2007, 68:1658-1667.

11. England JD, Gronseth GS, Franklin G, Carter GT, Kinsella LJ, Cohen JA, Asbury AK, Szigeti K, Lupski JR, Latov N, Lewis RA, Low PA, Fisher MA, Herrmann D, Howard JF, Lauria G, Miller RG, Polydefkis M, Sumner AJ: American Academy of Neurology; American Association of Neuromuscular and Electrodiagnostic Medicine; American Academy of Physical Medicine and Rehabilitation: Evaluation of distal symmetric polyneuropathy: the role of laboratory and genetic testing (an evidence-based review). Muscle Nerve 2009, 39:116-125.

12. Saporta AS, Sottile SL, Miller LJ, Feely SM, Siskind CE, Shy ME: Charcot-Marie-Tooth disease subtypes and genetic testing strategies. Ann Neurol 2011, 69:22-33.

13. Murphy SM, Laura M, Fawcett K, Pandraud A, Liu YT, Davidson GL, Rossor AM, Polke JM, Castleman V, Manji H, Lunn MP, Bull K, Ramdharry G, Davis M, Blake JC, Houlden H, Reilly MM: Charcot-Marie-Tooth disease: frequency of genetic subtypes and guidelines for genetic testing. J Neurol Neurosurg Psychiatry 2012, 83:706-710.

14. Plon SE, Eccles DM, Easton D, Foulkes WD, Genuardi M, Greenblatt MS, Hogervorst FB, Hoogerbrugge N, Spurdle AB, Tavtigian SV: IARC Unclassified Genetic Variants Working Group: Sequence variant classification and reporting: recommendations for improving the interpretation of cancer susceptibility genetic test results. Hum Mutat 2008, 29:1282-1291.

15. Bort S, Nelis E, Timmerman V, Sevilla T, Cruz-Martínez A, Martínez F, Millán JM, Arpa J, Vílchez JJ, Prieto F, Van Broeckhoven C, Palau F: Mutational analysis of the MPZ, PMP22 and Cx32 genes in patients of Spanish ancestry with Charcot-Marie-Tooth disease and hereditary neuropathy with liability to pressure palsies. Hum Genet 1997, 99:746-754.

16. Choi BO, Lee MS, Shin SH, Hwang JH, Choi KG, Kim WK, Sunwoo IN, Kim NK, Chung KW: Mutational analysis of PMP22, MPZ, GJB1, EGR2 and NEFL in Korean Charcot-Marie-Tooth neuropathy patients. Hum Mutat 2004, 24:185-186.

17. Mostacciuolo ML, Righetti E, Zortea M, Bosello V, Schiavon F, Vallo L, Merlini L, Siciliano G, Fabrizi GM, Rizzuto N, Milani M, Baratta S, Taroni F: Charcot-Marie-Tooth disease type I and related demyelinating neuropathies: Mutation analysis in a large cohort of Italian families. Hum Mutat 2001, 18:32-41.

18. Nicholson GA: Mutation testing in Charcot-Marie-Tooth neuropathy. Ann N Y Acad Sci 1999, 883:383-388.

19. Silander $K$, Meretoja $P$, Juvonen $V$, Ignatius J, Pihko $H$, Saarinen $A$, Wallden $T$, Herrgård E, Aula P, Savontaus ML: Spectrum of mutations in Finnish patients with Charcot-Marie-Tooth disease and related neuropathies. Hum Mutat 1998, 12:59-68.

20. Abe A, Numakura C, Kijima K, Hayashi M, Hashimoto T, Hayasaka K: Molecular diagnosis and clinical onset of Charcot-Marie-Tooth disease in Japan. J Hum Genet 2011, 56:364-368.

21. Antonidi T, Majumdar A, Burton-Jones S, Williams M: Inherited peripheral neuropathies: Genetic testing in the diagnostic laboratory [abstract]. Neuromuscul Disord 2011, 21:687-688.

22. Mersiyanova IV, Ismailov SM, Polyakov AV, Dadali EL, Fedotov VP, Nelis E, Löfgren A, Timmerman V, van Broeckhoven C, Evgrafov OV: Screening for mutations in the peripheral myelin genes PMP22, MPZ and Cx32 (GJB1) in Russian Charcot-Marie-Tooth neuropathy patients. Hum Mutat 2000, 15:340-347.

23. McCorquodale DS 3rd, Montenegro G, Peguero A, Carlson N, Speziani F, Price J, Taylor SW, Melanson M, Vance JM, Züchner S: Mutation screening of mitofusin 2 in Charcot-Marie-Tooth disease type 2. J Neurol 2011, 258:1234-1239.

24. Braathen GJ: Genetic epidemiology of Charcot-Marie-Tooth disease. Acta Neurol Scand 2012, 193:IV-22. 
25. Laššuthová $P$, Mazanec $R$, Vondráček $P$, Sišková D, Haberlová J, Sabová J, Seeman P: High frequency of SH3TC2 mutations in Czech HMSN I patients. Clin Genet 2011, 80:334-345.

26. Montenegro G, Powell E, Huang J, Speziani F, Edwards YJ, Beecham G, Hulme W, Siskind C, Vance J, Shy M, Züchner S: Exome sequencing allows for rapid gene identification in a Charcot-Marie-Tooth family. Ann Neurol 2011, 69:464-470.

doi:10.1186/1471-2350-14-94

Cite this article as: Østern et al.: Diagnostic laboratory testing for

Charcot Marie Tooth disease (CMT): the spectrum of gene defects in

Norwegian patients with CMT and its implications for future genetic

test strategies. BMC Medical Genetics 2013 14:94.

\section{Submit your next manuscript to BioMed Central and take full advantage of:}

- Convenient online submission

- Thorough peer review

- No space constraints or color figure charges

- Immediate publication on acceptance

- Inclusion in PubMed, CAS, Scopus and Google Scholar

- Research which is freely available for redistribution 